\title{
Sur une classe de polynômes qui se pré- sentent dans la théorie des fonctions cylindriques. (Deuxième partie.) *
}

\author{
(Par Niels Nielsen, à Copenhague.)
}

Dans cette deuxième partie de mes recherches sur le polynôme:

$$
R^{\mu, n}(x)=\underbrace{\leq \frac{n+1}{2}}_{s=0}(-1)^{s} \cdot \frac{(n-s) !}{s !}\left(\begin{array}{c}
\mu+n-s \\
n-2 s
\end{array}\right) \cdot\left(\frac{2}{x}\right)^{n-2 s}
$$

je me suis proposé de montrer comment les formules fondamentales de Lommer. nous conduiront à l'équation différentielle obtenue par M. HuRwitz pour notre polynôme. Après avoir démontré cette équation je déduis une nouvelle formule récursive très remarquable pour le calcul du polynôme $R$, formule qui fait voir clairement la nature flexible de notre polynôme, analogue à celle connue pour les fonctions et particulièrement pour les nombres de̊ JAcQues Bernouler.

Enfin, dans le dernier paragraphe on trouvera une application du polynôme susdit sur la résolution d'une équation aux différences finies linéaire et non homogène, résolution qui nous donnera un nombre de formules contenant les polynômes $R$ et les fonctions rationnelles $O^{n}(x)$ et $S^{n}(x)$ de M. Neumani et de Schrïrus.

(*) V. première partie dans ce journal, $3 .{ }^{\circ}$ S., t. V., p. $17 ; 1900$. 
332. Niels Nielsen: Sur une classe de polynômes qui se présentent

§ 1. Équation de M. Hurwitz. Formules récursives pour $R^{\mu, n}(x)$.

Pour démontrer que le polynôme $R^{\mu, n}(x)$ est intégrale particulière d'une équation différentielle linéaire et homogène prenons pour point de départ ces quatre formules de Lommer qui peuvent être trouvées dans la première partie $\left(^{*}\right)$ $\left[(9)\right.$, p. $22 ;\left(9_{a}\right)$, p. $23 ;(13)$ et $\left(13_{a}\right)$, p. 25], savoir :

$$
\begin{aligned}
& R^{\mu-1, n+1}(x)^{\mu+1, n-1}+R^{(x)}=\frac{2 \mu}{x} R^{\mu, n}(x), \\
& R^{\mu, n-1}(x)+R^{\mu, n+1}(x)=\frac{2(\mu+n+1)}{x} R^{\mu, n}(x), \\
& D_{x} \stackrel{\mu}{R, n}^{(x)}=-\frac{n}{x} R^{\mu, n}(x)+R^{\mu, n-1}(x)-R^{\mu+1, n-1}(x), \\
& D_{x} R^{\mu, n}(x)=\frac{2 \mu+n+2}{x} R^{\mu, n}(x)-R^{\mu, n+1}(x)-R^{\mu+1, n-1}(x) .
\end{aligned}
$$

Dans la première partie ces deux dernières formules se présentent défigurées par une faute d'impression, les derniers termes des seconds membres ayant en effet le signe + .

Les deux premières de nos formules donnent immédiatement cette autre:

$$
R(x)+R^{\mu+1, n}(x)=\frac{4 \cdots(\mu+-n+1)}{x^{2}} R^{\mu, n}(x)-R^{\mu+1, n-2}(x)-R^{\mu-1, n+2}(x),
$$

qui nous sera bien utile aussitôt. Pour démontrer la formule (1) il suffit d'exprimer, à l'aide de $(\alpha)$, chacune des fonctions figurant au premier membre et d'appliquer ensuite $(\beta)$.

Éliminant, à l'aide de $(0)$, la fonction $\frac{2 \mu}{x} R^{\mu, x}(x)$, figurant au second membre de $(\delta)$, on verra que $(\gamma),(\delta)$ se présentent sous cette forme plus élégante:

$$
D_{x} R^{\mu, n}(x)+\frac{n}{x} R^{\mu, n}(x)=R^{\mu, n-1}(x)-R^{\mu+1, n-1}(x)
$$

${ }^{*}$ ) Ce journal, $30^{\circ}$ sórie, t. V, fasc. I, p. 17-31, 1900. Je cite toujours cette premièro partie en écrivant dans une parenthèse le numéro de la formule en question et le pare dı volume susdit où elle peut être trouvée. 


$$
D_{x} R^{\mu, n}(x)-\frac{n+2}{x} R^{\mu, n}(x)=R^{\mu-1, n+1}(x)-R^{\mu, n+1}(x) .
$$

Posant encore dans (2) $\mu-1$ au lieu de $\mu$ et $n+2$ au lieu de $n$, on aura, à l'aide de $\left(2_{a}\right)$, cette formule remarquable:

$$
D_{x}\left(R^{\mu, n}(x)-R^{\mu-1, n+2}(x)=\frac{n+2}{x}\left(R^{\mu, n}(x)+R^{\mu-1, n+2}(x)\right),\right.
$$

ou bien, après avoir posé $\mu+1$ au lieu de $\mu$ et $n-2$ au lieu de $n$ :

$$
D_{x}\left(R(x)-R^{\mu+1, n-2}(x)\right)=-\frac{n}{x}\left(R^{\mu, n}(x)+R^{\mu+1, n-2}(x)\right) .
$$

Démontrons maintenant comment les cinq formules de (i) à $\left(3_{a}\right)$ nous conduiront à l'équation différentielle cherchée.

En effet, différentions par rapport à $x$ la formule (2), nous aurons, en exprimant, à l'aide de $\left(2_{a}\right)$, les dérivées obtenues au second membre:

$$
y^{\prime \prime}-\frac{1}{x} y^{\prime}-\frac{n(n+2)}{x^{2}} y=R^{\mu-1, n}(x)-2 R^{\mu, n}(x)+R(x),
$$

où $y$ désigne le polynôme $R(x)$. Cela posé, appliquons (1), nous aurons:

$$
y^{\prime \prime}-\frac{1}{x} y^{\prime}-\left(\frac{n(n+2)+4 \mu(\mu+n+1)}{x^{2}}-2\right) y=-R^{\mu-1, n+2}(x)-R^{\mu+1, n-2)}(x) \cdot(\varepsilon)
$$

Différentions de nouveau par rapport à $x$, nous obtiendrons, en vertu de $(3),\left(3_{\alpha}\right)$, pour le second membre cette expression:

$$
-2 y^{\prime}+\frac{2}{x} y+\frac{n+2}{x}\left(R^{\mu-1, n+2}(x)+R_{(x)}^{\mu+1, n-2}\right)-\frac{2 n+2}{x} R_{(x)}^{n+1, i-2}
$$

d'où, à l'aide de $(\varepsilon)$ :

$$
\begin{gathered}
y^{\prime \prime}+\frac{n+1}{x} y^{\prime \prime}+\left(4-\frac{(n+1)(n+2)+4 \mu(\mu+n+1)-1}{x^{2}}\right) y^{\prime}+ \\
+\left(\frac{2 n+2}{x}-\frac{n^{2}(n+2)+4 n \mu(\mu+n+1)}{x^{2}}\right) y= \\
=-\frac{2 n+2}{x} R^{\mu+1, n}(x) .
\end{gathered}
$$

Enfin, différentions encore une fois par rapport à $x$, nous aurons, a l'aide de $\left(3_{a}\right)$, pour le second membre cette expression:

$$
-\frac{2 n+2}{x} y^{\prime}-\frac{2 n(n+1)}{x^{2}} y-\frac{2(n+1)(n-1)}{x^{2}} R^{u+1, n)},^{2}
$$


334 Niels Nielsen: Sur une classe de polynômes qui se présentent

ce qui donnera finalement, en vertu de $(\zeta)$ :

$$
\begin{gathered}
y^{\mathrm{IV}}+\frac{2}{x} y^{\prime \prime \prime}+\left(4-\frac{2(n+1)^{2}+4 \mu(\mu+n+1)-1}{x^{2}}\right) y^{\prime \prime}+ \\
+\left(\frac{8}{x}+\frac{2(n+1)^{2}+4 u(\mu+n+1)-1}{x^{3}}\right) y^{\prime}+ \\
+\frac{n(n+2)(n+2 \mu)(n+2 \mu+2)}{x^{4}} y=0
\end{gathered}
$$

et voilà l'équation différentielle que M. Hurwitz $\left(^{*}\right)$ a énoncée sans démonstration dans son fondamental Mémoire sur les zéros de la fonction $J^{\mu}(x)$. La fonction $g_{m}$ de M. HuRwirz s'exprime à l'aide de $R^{\mu, n}(x)$ de la manière suivante :

$$
g_{n i}^{\mu}(z)=\left(i \sqrt{z}^{m}\right) \cdot R(2 i \sqrt{z})
$$

La formule $\left(3_{a}\right)$ qui nous a été si utile pour la démonstration de (4) donnera, à l'aide de $(\gamma)$ et en remarquant que les deux polynômes $R^{r, 0}(x)$ et $R(x)$ sont indépendantes de $x$ tous les deux, cette autre formule:

$$
R^{\mu+1, n-1}(x)-R^{\mu, n-1}(x)=\frac{2}{x} \cdot \underbrace{\sum_{2}^{\frac{n}{2}}}_{p=1}(n-2 p+1) R^{\mu+p, n-2 p}(x)
$$

qui ne peut pas être déduite à l'aide des formules analogues développées dans la première partie (pp. 26, 27).

Nous avons encore à démontrer une formule, nouvelle que je sache, qui permettra d'exprimer le polynôme $R(x)$ à l'aide des polynômes du même genre et dont les indices entiers sont égaux à $\frac{n}{2}$ au plus. A cet égard prenons pour point de départ la formule:

$$
\frac{J^{\mu}(x)}{r !}=\sum_{s=0}^{s=\bar{\Sigma}} \frac{(-1)^{r-s}}{(r-s) !}\left(\begin{array}{c}
u-r+s-1 \\
s
\end{array}\right)\left(\frac{2}{x}\right)^{s} J^{\mu-2 r+s}(x),
$$

(*) Mathematische Annalen, t. XXXIII, p. 251, note; 1889. (Du reste, l'intégrale complète de l'équation (4) pent s'écrire sous cette forme:

$$
y=x\left(a J^{\mu}(x) J^{\mu+n+1}(x)^{\mu}+b J^{\mu}(x) Y^{\mu+(x+1}+c Y^{\mu}(x) J^{\mu}(x)+d Y^{\mu}(x) Y^{\mu+n+1}(x)\right),
$$

ce qui s'accorde bien avec la formule fondamentale de Lommes, voir $(\gamma)$, p. 7.) 
où $\gamma^{*}$ désigne un entier non négatif $\left({ }^{*}\right)$. Posons maintenant $\mu+m$ au lieu de $\mu$, nous aurons, d'après une formule démontrée dans la première partie $[(10)$, p. 24], cette formule remarquable:

$$
\frac{R^{\mu, n}(x)}{r !}=\sum_{s=0}^{s=\mathbf{y}^{r}} \frac{(-1)^{r \sim s}}{(r-s) !}\left(\begin{array}{c}
\mu+m-r-s \\
s
\end{array}\right)\left(\frac{2}{x}\right)^{s} R^{\mu, m-2 r+s}(x),
$$

où nous avons posé $\mu+1$ au lieu de $\mu$. Il est évident qu'une foule de formules plus particulières peut être déduite de (6). Supposons par exemple que le positif entier $m$, soit égal à $2 r$ ou à $2 v+1$, les formules correspondantes se présentent sous forme très élégante.

\section{§ 2. RÉSOLUTION DE L'ÉQUATION AUX DIFFt́RENCES FINIES}

$$
G^{n-1}(x)+G^{n+1}(x)=\frac{2 n}{x} G^{n}(x)+\frac{2}{x} g^{n}(x), \quad n \text { ENTIER. }
$$

En supposant que $G^{\mu}(x)$ satisfasse à cette équation linéaire aux différences finies :

$$
G^{\mu-1}(x)+G^{\mu+1}(x)=\frac{2 \mu}{x} G^{\mu}(x)+\frac{2}{x} g^{\mu}(x)
$$

où $g^{\mu}(x)$ désigne une fonction donnée de $x$ et de $\mu$, nous aurons $[(14)$, p. 26]:

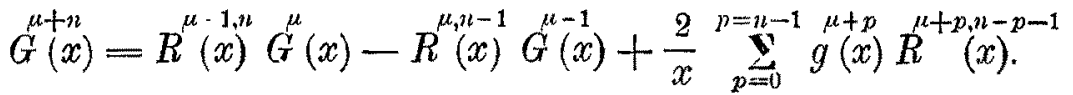

Remarquant en outre que $\cos \mu \pi \cdot G^{-\mu}(x)$ satisfait aussi à une équation déduite de $(\alpha)$ en y remplacant $g^{\mu}(x)$ par $-\cos \mu \pi \cdot g(x)$, on aura encore, après avoir changé le signe de $\mu$ et supprimé le facteur commun $\cos \mu \pi$, cette autre formule analogue à $(B)$ :

$$
\left.\begin{array}{c}
(-1)^{n} G(x)=R(x) G^{\mu-n}(x)+R(x) G^{-\mu+1, n} G^{\mu}(x)- \\
-\frac{2}{x} \sum_{p=0}^{p=n-1}(-1)^{p} g^{\mu-p}(x) R_{(-\mu+p, n-p-1}^{(x) .}
\end{array}\right\}
$$

(*) Ce journal, 3. ${ }^{\circledR}$ série, t. VI, p. 94; 1901. 
336 Niels Nielsen: Sur une classe de polynômes qui se présentent

Inversement, la formule $(\beta)$ au $\S 1$ montrera immédiatement que la fonction $G \stackrel{\mu \pm n}{(x)}$ satisfait à l'équation obtenue de $(\alpha)$ en y posant $\mu \pm n$ au lieu de $\mu$, pourvu que $G^{\mu}(x)$ satisfasse à ( $\alpha$ ) elle-même.

$I l$ est bien remarquable que le second membre de $(B)$ doit étre une fonction de $\mu \pm n$.

Supposons maintenant $\mu=1$ et mettons $n-1$ au lieu de $n$, nous aurons, en vertu de $(\beta)$ :

$$
G^{n}(x)=R^{0, n-1}(x) C^{1}(x)-R^{1, n-2}(x) G^{0}(x)+\frac{2}{x} \cdot{ }_{p=0}^{p=n-2} g(x) \cdot R^{p+1, n-p \ldots 2}
$$

tandis que $\left(\beta^{\prime}\right)$ donnera pour $\mu=-1$ :

$$
\left.\begin{array}{c}
(-1)^{n} G^{-n}(x)=R^{0, n-1}(x) G^{1}(x)-R^{1, n-2}(x) G^{0}(x)- \\
-\frac{2}{x} \cdot \sum_{p=0}^{p=n-1}(-1)^{p} g^{-p}(x) R^{p, n-p-1}(x),
\end{array}\right\}
$$

car nous aurons toujours:

$$
G^{-1}(x)=\frac{2}{x} g^{0}(x)-G^{1}(x) .
$$

Cela posé, nous avons démontré cette proposition:

Les formules $(7),\left(7_{a}\right)$ nous donnent la solution complète de l'équation aux différences finies $(\alpha)$, pourvu que $\mu$ soit égal à un nombre entier. $G^{0}(x)$ et $G^{1}(x)$ sont deux fonctions arbitraires de $x$.

Dans mon Mémoire : Évaluation nouvelle etc. $\left(^{*}\right)$ j'ai donné, à l'aide des sẻries neumanniennes de première espèce, la solution d'une équation particulière de la forme $(\alpha)$.

Posons par exemple

$$
g^{n}(x)=2 \cos ^{2} \frac{n \pi}{2}, \quad G^{0}(x)=0, \quad G^{1}(x)=\frac{2}{x},
$$

nous retrouvons la fonction ${ }^{n} S^{n}(x)$ de SCHLÄ̈LI exprimée à l'aide des polynômes $R[(20)$, p. 28]. Or, en se rappelant la formule

$$
O^{n}(x)=\frac{2 n}{x} S^{n}(x)+\frac{\cos ^{2} \frac{n \pi}{2}}{x},
$$

(*) Ce journal, 3. ${ }^{\mathrm{e}}$ série, t. VI, p. 106; 1901. 
on retombe par là dans les deux formules qui se présentent sous forme masquée dans le beau livre de M. J.-H. GraF (*).

En remarquant que $J^{n}(x)$ et $Y^{n}(x)$ représentent deux solutions, indépendantes entre elles, de l'équation homogène qui correspond à $(\alpha)$, on trouvera aisément les formules $(7),\left(7_{a}\right)$ aussi à l'aide de la méthode générale de $\mathrm{L}_{A}$ GRANGE $\left(^{* *}\right)$ et en appliquant cette formule fondamentale de LoMmer:

$$
Y^{\mu}(x) J^{\mu+\mu}(x)-Y^{\mu+n}(x) J^{n}(x)=\frac{2}{x} R \cdot(x)
$$

où $n$ désigne un positif entier.

Désignons maintenant par $G_{1}^{n}(x)$ une solution, différente de $G^{n}(x)$, nous aurons une équation de cette forme $[(22)$, p. 29]:

$$
G^{n}(x)-G_{1}^{n}(x)=a(x) J^{n}(x)+b(x) Y^{n}(x),
$$

où $a(x), b(x)$ sont deux fonctions convenables de $x$ mais indépendantes de Or, cette formule peut être vérifiée aisément à l'aide de $(7),\left(7_{a}\right)$. En effe', posons dans (8) $n=0$ et $n=1$, nous aurons, en vertu de $(y)$ :

$$
\left.\begin{array}{l}
\frac{2}{x} a(x)=\left(G^{1}(x)-G_{1}^{1}(x)\right) Y^{0}(x)-\left(G^{0}(x)-G_{1}^{0}(x)\right) Y^{1}(x), \\
\frac{2}{x} b(x)=\left(G^{0}(x)-G_{1}^{0}(x)\right) J^{1}(x)-\left(G^{1}(x)-G_{1}^{1}(x)\right) J^{0}(x),
\end{array}\right\}
$$

ce qui donnera, à l'aide de $(\gamma)$ :

$$
\left.\begin{array}{c}
a(x) J^{\prime \prime}(x)+b(x) Y^{n}(x)=R \stackrel{0, n-1}{(x)}\left(G^{1}(x)-G_{1}^{1}(x)\right)- \\
-R^{1, n-2}(x)\left(G^{0}(x)-G_{1}^{0}(x)\right)
\end{array}\right\}
$$

et voilà précisément la formule qu'il fallait de démontrer.

Supposons que $a(x), b(x)$ soient deux fonctions arbitraires de $x$ mais indépendantes de $n$, le second membre de (8) représente la solution complète de l'équation homogène qui correspond à $(\alpha)$. Or, mettons:

$$
a(x)=\frac{x}{2} Y^{k}(x), \quad b(x)=-\frac{x}{2} J^{k}(x)
$$

(*) Einleitung in die Theorie der Bessel'schen F'unctionen, fasc. II, p. 41; Berne 1900.

(**) Voir par exemple MarkorF: Differenzentechnung, p. 15I; Leipsic 1896.

Annali di Matematica, Serie III, tomo VI. 
338 Niels Nielsen: Sur une clusse de polynômes qui se présentent

$k$ désignant un nombre entier fixe indépendant de $n$, nous aurons, en vertu de $(\gamma)$ :

$$
a(x) J^{n}(x)+b(x) Y^{n}(x)=R \stackrel{k, n-k-1}{(x)}
$$

de sorte que cette fonction définie pour les valeurs négatives de $n-k-1$ à l'aide de la formule $\left[\left(6_{a}\right), \mathrm{p} .21\right]$ :

$$
R^{\mu,-n-1}(x)=(-1)^{n} R^{-\mu, n-1}(x)=-R^{\mu-n, n-1}
$$

représente aussi une solution de notre équation homogène, résultat qui s'accorde bien avec la forme générale d'une telle solution.

J'ai traité cette question d'une manière si détaillée pour corriger une faute d'écriture qui a echappée à mon attention dans les épreuves de la première partie. La remarque faite au p. 23 nie simplement cette propriété de $R \stackrel{k, n-k-1}{(x)}$

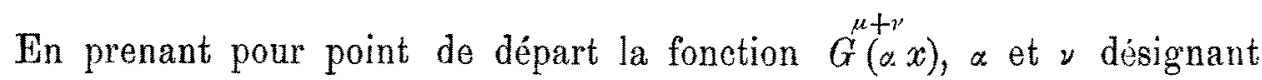
deux quantités finies quelconques, on peut beaucoup généraliser la formule $(\beta)$ de ce paragraphe. En effet, la fonction susdite satisfait à une équation de la forme $(\alpha)$, pourvu que $g^{\mu}(x)$ soit remplacée par

$$
\left(\frac{\nu+\mu}{\alpha}-\mu\right) G(\alpha x)+\frac{1}{\alpha} g^{\mu+\nu}(\alpha) \text {. }
$$

Cela posé, appliquons de nouveau $(\beta)$, nous aurons, après avoir posé $y$ au lieu de $\alpha x$ et $\nu-\mu$ au lieu de $\nu$, cette formule générale:

$$
\begin{aligned}
& G^{\nu+1}(y)=R^{\mu-1, n}(x) G^{\nu}(y)-R^{\mu, n-1}(x) G^{\nu-1}(y)+ \\
& +\frac{2}{y} \cdot \sum_{p=0}^{p=n-1} g^{r+p}(y) R^{\mu+p, n-p-1}(x)^{-1}+ \\
& +2 \sum_{p=0}^{p=-n-1}\left(\frac{v+p}{y}-\frac{\mu+p}{x}\right) G(y) R^{\mu+p+p, n-p-1}
\end{aligned}
$$

qui est bien remarquable à cause des quatre variables qu'elle contient. Il est clair que cette formule n'est autre chose qu'une conséquence des propriétés fondamentales des polynômes $R$, de sorte qu'elle dénonce de nouveau la nature Hexible des polynômes susdits. Néanmoins, une démonstration de (10) par un calcul direct semble être un peu longue. 
Or, il est clair que la formule (10) pourra donner une foule d'autres ou en spécialisant les quantités $\mu, \nu, x, y$ ou en introduisant des fonctions particulières au lieu de $G$. C'est par ce point de vue que j'ai déduit, dans la première partie, une suite de formules contenant les polynômes $R$, en supposant $G$ égal à une fonction cylindrique.

Voici maintenant quelques autres formules particulières déduites de (10): 1. ${ }^{\circ} x=\infty$, posant

$$
H^{n}(x)=\frac{n}{2 x} G^{n}(x)+\frac{1}{2 x} g \stackrel{n}{n}(x),
$$

et appliquant la formule:

$$
R(\infty)=\cos \frac{k \pi}{2}
$$

on aura, après avoir écrit $x$ au lieu de $y$ et posé $\nu=0$ :

$$
G^{n}(x)=4 \cdot \sum_{p=1}^{p=n-1} \sin \frac{n-p}{2} \pi \cdot H^{p}(x)+\sin \frac{n \pi}{2} G^{1}(x)+\cos \frac{n \pi}{2} G^{0}(x),
$$

formule qui pent être déduite aussi à l'aide de celle:

$$
G^{n-1}(x)+G^{n+1}(x)=4 H^{n}(x)
$$

qui est une conséquence immédiate de $(\varepsilon)$. Pour les fonctions $O^{n}(x), S^{n}(x)$ on retrouve par là une formule observée par M. GRAF (*) pour la première fois, d'après ce que je sais.

2. ${ }^{\circ} \quad x=-y, \nu=1, \mu=1$, nous aurons, après avoir écrit $x$ au lieu de $y$ :

$$
\begin{aligned}
& (-1)^{n-1} G^{n}(x)=G^{1}(x) R^{0, n-1}(x)+G^{0}(x) R(x)+ \\
& +\frac{2}{x} \cdot \sum_{p=0}^{p=n-1}(-1)^{p}\left(g^{p}(x)+2 p G^{p}(x)\right) \cdot R^{p, n-p-1}(x),
\end{aligned}
$$

ou bien, en vertu de $(\varepsilon)$ :

$$
\left.\begin{array}{l}
(-1)^{n-1} G^{n}(x)=G^{1}(x) R^{0, n-1}(x)+G^{0}(x) R^{1, n-2}(x)- \\
-\frac{2}{x} \cdot \sum_{p=0}^{p=n-1}(-1) p\left(4 x H^{p}(x)-g^{p}(x)\right) R^{p, n-p-1}(x) .
\end{array}\right\}
$$

(*) Loc. cit., fasc. II, p. 28 . 
Mettons dans (12) $S^{n}(x)$ au lieu de $G^{n}(x)$, nous aurons, en vertu de [(20;, p. 281 :

$$
\begin{gathered}
S^{2 n}(x)=\frac{2}{x} \cdot \sum_{p=1}^{p=2 n-1}(-1)^{p-1} \cdot p \cdot S^{p}(x) R^{p, 2 n-p-1}(x), \\
O^{2 n}(x)=\frac{1}{2 x}+\frac{1}{2 x} \cdot \sum_{p=1}^{p=2 n-1}(-1)^{p-1} \cdot p \cdot S^{p}(x) R^{p, 2 n-p}(x),
\end{gathered}
$$

tandis que $\left(12_{a}\right)$ donnera de mêrne:

$$
\begin{aligned}
& S^{2 n+1}=4 \cdot \sum_{p=0}^{p=2 n}(-1)^{p} O^{p}(x) R^{p, 2 n-p}(x) \\
& O^{2 n+1}(x)=4 \cdot \sum_{p=0}^{p=2 n}(-1)^{p} O^{n}(x) R^{p, 2 n-p+1}(x)
\end{aligned}
$$

où l'accent après le signe $\Sigma$ indique qu'il faut prendre la moitié du terme qui correspond à $p=0$.

On trouvera évidemment huit formules analogues contenant les deux fonctions $\Xi_{1}^{n}(x)$ et $\Xi_{2}^{n}(x)$ qui sont réelles pourru que $x$ le soit et qui peuvent être définies à l'aide de la formule:

$$
\mathfrak{S}^{n}(x)=e^{-i x}\left(\mathfrak{S}_{1}^{n}(x)+i \mathfrak{S}_{2}^{n}(x)\right)
$$

$\varsigma^{n}(x)$ désignant la fonction que j'ai introduite dans la première partie (p. 28) et que j'ai étudiée dans mon Mémoire: Evaluation nouvelle etc. $(*)$

Copenhague, le 18 janvier 1901.

(*) Ge journal, 3. ${ }^{\circ}$ série, t. VI, p. 82 . 